\title{
Assessment of Performance of Pharmaceutical Firms making Drugs for Specific Diseases Prevalent in India: \\ A Data Envelopment Analysis Approach

\author{
'Sumanta Das, ${ }^{2}$ G.N Patel \\ ${ }^{1}$ Research Scholar, Birla Institute of Management Technology (BIMTECH), Greater Noida, U.P, India \\ E-mail: sumantadas@hotmail.com \\ ${ }^{2}$ Professor,Birla Institute of Management Technology (BIMTECH), Greater Noida, U.P, India \\ E-mail: gn.patel@bimtech.ac.in
}

\begin{abstract}
There exists a lot of diversity in Indian pharmaceutical environment in various aspects. We have applied Delphi Technique to arrive at specific prevalent diseases in India and identified 24 pharmaceutical firms making drugs for these diseases. We used data envelopment analysis (DEA) approach to find out the efficiency of firms and peers of inefficient firms. Tobit regression has been used to get the determinants of efficiency.
\end{abstract}

\section{Indexing terms/Keywords}

Data envelopment Analysis (DEA), Pharmaceutical firms, Delphi technique, BCC model, Tobit regression.

\section{Academic Discipline And Sub-Disciplines}

Management Science

\section{SUBJECT CLASSIFICATION}

Data Envelopment Analysis

\section{TYPE (METHOD/APPROACH)}

Qualitative approach used for assessment of opinions through Delphi technique and Quantitative inferential approach used through DEA.

\section{Council for Innovative Research}

Peer Review Research Publishing System

Journal: International Journal of Management \& Information Technology

Vol. 6, No. 2

editor@cirworld.com

www.cirworld.com, member.cirworld.com 


\section{INTRODUCTION}

India is rapidly growing to become a major and vibrant economic power, in spite of the wide disparities within its social milieu. There is increase, also in population of higher income group, who do not hesitate to afford branded costlier drugs in preference to cheaper alternatives (FICCl - Ernst \& young study, 2010). India is now becoming a lucrative destination for global pharmaceutical giants. It is estimated that India may become a US\$ 8 billion drug market by 2015 for the MNCs. Domestic pharmaceutical market is expected to touch USD 21 billion by 2015, from 7.1 billion in 2007 (www.moneyexpress.com/ business/33701.txthtml).

The management of pharmaceutical business includes marketing, quality assurance, $R \& D$, finance, operations, wholesale and drug store management. The application of drug laws and/or intellectual property rights will be of necessary use for those in pharmaceutical business. Recent developments in pharmaceutical sector in India reveal many mergers and acquisitions (M\&A) taking place, resulting in shooting up of revenues of the business. The Indian companies are getting involved in this M\&A space as there is growing domestic market for cheaper versions of patent drugs. (i.e. generic drugs) which also will be able to serve developed markets abroad. Unlike in past, the Multi National Companies (MNCs) pharmaceutical companies are clearly shifting their strategies:- from giving out manufacturing contracts to Indian companies to owning them. Multi National Companies (MNCs) are attracted by the emerging markets in Indian scenario for generic drugs. This trend is noticed in the strategy of companies such as Pfizer, GSK, Abott and Sanofi. The pharmaceutical market has witnessed a strong double - digit growth ranging from $13.5 \%$ to $17 \%$ over the past four years (with the exception of the year 2008 when growth was relatively low at $10 \%$ ). So for those MNCs facing sluggish sales in their home markets, even a market share of 2-3\% in India could be attractive. (Mergers \& Acquisitions in Pharmaceutical industry- News report Times of India:16.02.2011). Some of M\&A in pharmaceutical industries are shown in Table-1.

The tendency of Indian pharmaceutical majors being bought by MNCs orients them away from Indian markets, and it may have adverse effect on drug pricing and affordability on the part of the average Indian consumer. In last four years, at least six big acquisitions of this kind took place.

Table-1 Mergers and Acquisitions in Pharmaceutical Industries:

\begin{tabular}{|l|l|l|l|}
\hline Year & Indian Company taken over & Foreign Buyer & Deal size \$ Million \\
\hline June'08 & Ranbaxy Lab & Daiichi Sankyo,Japan & 4600 \\
\hline July'08 & Shantha Biotech & Sanofi Aventis, France & 783 \\
\hline Dec'09 & Orchid & Hospira, USA & 400 \\
\hline Aug'06 & MatrixLab & Mylan Inc. & 736 \\
\hline May'10 & Piramal Healthcare & Abott, USA & 3720 \\
\hline Dec'10 & Paras Pharma & Reckitt Benckiser, USA & 726 \\
\hline
\end{tabular}

(source: Mergers \& Acquisitions in Pharmaceutical industry- News report Times of India :16.02.2011: "Pharma sector injects fresh life into $M \& A$ space)

The biosimilar opportunity is also big, that big pharmaceutical giants don't want to miss it. Merck has established a subsidiary and Pfizer has tied up with Biocon. A biosimilar is approximate copy of a patented biological drug. It is approximate copy because it is hard or impossible to make an exact copy of a biological at the moment. While generic drugs have made a big dent in the market, regulators had not allowed generics companies to make off-patent biological. They are relaxing their attitudes due to the high cost of patented biologicals. As in the generics, Indian companies are looking at a large global market in biosimilars. Big opportunity for Indian firms as several blockbusters goes off patent during year $2017-2024$. The size of the biosimilars market by 2020 is expected to be $\$ 11$ Billion (: Economic Times, 1 st March 2012).

In view of above discussed scenario, it is quite obvious that costly drugs, which are ordinarily beyond the reach of average Indian consumer and which are manufactured by pharmaceutical companies including MNCs, are still reigning the market and enjoying their share in a tough competitive market. Nevertheless, keeping larger public good in mind, it will be in the best interest of even these companies to tailor down their costs to meet needs of consumer,without any compromise on quality. Keeping wide spectrum of prevalent diseases in India in view, analysis may be conducted to assess the firms in making available of vital drugs on basis of certain criteria such as:

1. Generic formulations of single drugs or generic formulations of scientifically acceptable and rational combinations.

2. Assessment of these firms spends on R\&D which eventually increases the cost of medicine.

3. Analyze if efficient firms are cost efficient. As cost efficient firm can provide drugs at affordable price.

4. Increase in marketing efforts by the firms supposedly improves the accessibility of drugs.

By such a study, it may be possible to understand clear picture of comparative assessment of the market place economics of drugs and of different firms at large to help the consumers. Like with improved cost efficiency may lead to scope of reduction of price.

\section{Previous Studies}

A number of studies have been published on different aspects pertaining to pharmaceutical firms. Some of the work done by authors throws lot of insights on pharmaceutical industry scenario in Indian context. Kotwani et.al. (2007) studied on prices \& availability of common medicines at six sites in India. Sengupta (2010) worked on National Health System in India with regards to access to Health Care and Medicines. His work provided detail framework and policy pertaining to Indian pharmaceutical industry. 
Sampath (2005) provided studies on "Economic Aspects of Access to Medicines after 2005: Product Patent Protection and Emerging Firm Strategies in the Indian Pharmaceutical Industry". Dhar \& Gopukumar (2006) provided detailed information on Post-2005 Trade Related Aspects of Intellectual Property Rights (TRIPS) scenario in patent protection in the pharmaceutical sector in particular to generic pharmaceutical industry in India. Kiran Ravi \& Mishra Sunita (2011) also provided a post TRIPS analysis on pharmaceutical companies.

Some of the papers published based on financial statement analysis of the firms. We are focusing mainly on those studies that use frontier analysis or DEA to gauge performance of pharmaceutical firms.

Pannu et.al. (2010) studied on aspect of Innovation on performance of Indian Pharmaceutical Industry. Malhotra \& Malhotra (2008) used DEA where it employs relative efficiency, a concept enabling comparison of companies with a pool of known efficient companies. The inputs considered are "days of sales outstanding, days cost of goods, sold in inventory, total debt/equity. The outputs considered are "cash flow per share, return on equity, return on assets, return on invested capital, inventory turnover, interest rate coverage, quick ratio". Saranga (2007), Saranga and Phani (2004) used DEA to study the performance of pharmaceutical companies in order to determine the best practices in the Indian Pharmaceutical industry. Mazumdar et.al. (2010) used DEA efficiency score of a firm which provides an assessment of its performance based on measurement of output and input efficiencies for Indian pharmaceutical firms.

\section{Identification of prevalent disease in India}

Delphi Technique is used to arrive at specific prevalent diseases in India. Delphi technique used to form consensus after opinions from experts for specific prevalent diseases in Indian context. Study on these firms was arrived based on drugs (generic names) and older (Innovator) drugs, specific against the diseases, available in market.

The Delphi technique was developed during the 1950s by workers at the RAND Corporation while involved on a U.S. Air Force sponsored project. The aim of the project was the application of expert opinion to the selection - from the point of view of a Soviet strategic planner - of an optimal U.S. industrial target system, with a corresponding estimation of the number of atomic bombs required to reduce munitions output by a prescribed amount. More generally, the technique is seen as a procedure to "obtain the most reliable consensus of opinion of a group of experts . . . by a series of intensive questionnaires interspersed with controlled opinion feedback" (Dalkey \& Helmer,1963). Delphi technique was earlier used for futuristic study, but currently it is being used extensively in social sciences to arrive at a consensus by a panel of experts (Chia \& Sandford, 2007).

We used Delphi technique with a panel of seven judges, working at a distance, with all communication by email through a moderator. There are two separate stages, shortlisting and judging, each consisting of an initial round that elicits panelists comments on the entries, followed by three rounds in which panelists nominated their preferred entries. The number of rounds depends on how quickly a consensus emerges.

Panelists sent their responses to the author who was the moderator, which was collated and circulated to them anonymously after each round, as the basis for the next round. The panelists have at each stage a full record of what comments and nominations other panelists have made, but they do not know who made which comment or voted for which entry. Nor do they know the final result, till the same was disclosed after the consensus which was reached after 3 rounds for the widely prevalent diseases in Indian context fit for the studies. The following diseases and the relevant drugs are being considered for study out of the exhaustive list of widely prevalent and emerging diseases in Indian population. This study is further based on following diseases supported by expert opinion of Medical professionals as selected by the process cited above:-

Communicable diseases:

\section{Tuberculosis}

The treatment of Tuberculosis, world over, is based on WHO guidelines, according to which the first line drugs (categories I, II \& III) for routine cases and second line drugs, which are given in drug-resistant cases i.e multi drug resistant tuberculosis (MDRTB) (source: 1. TB-A clinical manual for South -east Asia: Harries et.al., World Health Organization (WHO), Geneva,1997, 2. Guidelines for the management of drug- resistant Tuberculosis Crofton et.al.WHO, Geneva,(1997)

The treatment of the disease is done based on the category of patient affected by the infection. The treatment can be done based on administration of First line Drugs or 2nd Line drugs for MDRTB, as the case may be. The list of I st Line drugs and manufacturers is provided in Table -2. Similarly for 2nd line drug is illustrated in Table-4.

Human immunodeficiency virus infection / acquired immunodeficiency syndrome (HIV/AIDS)

Anti Retroviral drugs used for treatment of HIV-AIDS is provided in Table 3.

Non communicable diseases

\section{Diabetes mellitus}

The treatment for Diabetes based on the severity is done through administration of oral hypoglycemic agents as Shown in Table-5. The treatment done through administration of Biguanides and its combinations are provided in Table-7. Table-6 provides the list of companies into different types of Insulin used for treatment of Diabetes. 
Table-2 - 1st line drugs for Tuberculosis and Medicine Company name

\begin{tabular}{|c|c|c|c|c|c|c|c|c|c|}
\hline \multirow{2}{*}{\begin{tabular}{|l|} 
Disease \\
Medicine
\end{tabular}} & \multicolumn{9}{|c|}{ Tuberculosis (Ist line drugs) } \\
\hline & $\begin{array}{l}\text { Rifamycin } \\
\text { (RIFA) }\end{array}$ & $\begin{array}{l}\text { Isonicotonic acid } \\
\text { Hydrazide (INH) }\end{array}$ & $\begin{array}{l}\text { Pyrazinamide } \\
\text { (PZA) }\end{array}$ & $\begin{array}{l}\text { Etham butanol } \\
\text { (ETB) }\end{array}$ & $\mathrm{ETB}+\mathrm{INH}$ & RIFA + INH & $\begin{array}{l}\text { RIFA + INH } \\
+ \text { PZA }\end{array}$ & $\begin{array}{l}\text { RIFA + INH +PZA } \\
+ \text { ETB }\end{array}$ & $\begin{array}{l}\text { Streptomycin } \\
\text { (injectible) }\end{array}$ \\
\hline \multirow[t]{24}{*}{$\begin{array}{l}\text { Company } \\
\text { name }\end{array}$} & Aristo & Park Davis & Novartis & Lupin & Lupin & Unichem & Novartis & Novartis & $\begin{array}{l}\text { SPPL-Ethical } \\
\text { Div }\end{array}$ \\
\hline & Lark Labs & Albert David & Macleods & Macleods & Plethico & Ovearseas HC & Unichem & Larks Lab & \\
\hline & Lupin & IPCA & Lark Labs & RPG lifesciences & Cadilla Pharma & BiologicalE & BiologicalE & Lupin & \\
\hline & Macleods & Pfizer & Taurus & Panacea Biotech & Le Sante & Merind & Platheco & Unichem & \\
\hline & Plathico & Macleods & Lasante & Samarth Pharm & Themis & Aristo & Merind & BiologicalE & \\
\hline & Concepts & & Overseas HC & Concepts & & Alkem & Aristo & Merind & \\
\hline & IDPL & & PCI & Emcure & & Zydus Cadilla & Alkem & Alkem & \\
\hline & Le Sante & & Emcure & Pharmed & & Emcure & Emcure & Zydus Cadilla & \\
\hline & Overseas HC & & Aristo & Plethico & & IDPL & Lark Labs & Macleods & \\
\hline & Biochem & & Le Sante & Larks Labs & & Lark Labs & IPCA & Platheco & \\
\hline & Zydus Cadilla & & Pharmed & Le Sante & & IPCA & Macleods & Le Sante & \\
\hline & Novartis & & Lupin & Overseas HC & & Biochem & Le Sante & Suniz & \\
\hline & Themis & & Novartis & Taurus Labs & & Pharmed & Lupin & Overseas HC & \\
\hline & Taurus labs & & Themis & Themis & & Macleods & Suniz & Indoco & \\
\hline & & & Taurus & & & Platheco & Overseas SC & Concepts & \\
\hline & & & & & & GSK & Concept & Themes & \\
\hline & & & & & & & Indoco & Rextar & \\
\hline & & & & & & & Aventis & Wockhardt & \\
\hline & & & & & & & Kresp & GSK & \\
\hline & & & & & & & Novartis & & \\
\hline & & & & & $=$ & & Pharmed & & \\
\hline & & & & 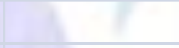 & & & Rextar & & \\
\hline & & & & & & 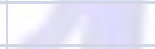 & Themes & & \\
\hline & & & & & & & GSK & & \\
\hline
\end{tabular}

(Source: Industry Drug Review)

Table-3. Antiretroviral drug for HIV/AIDS

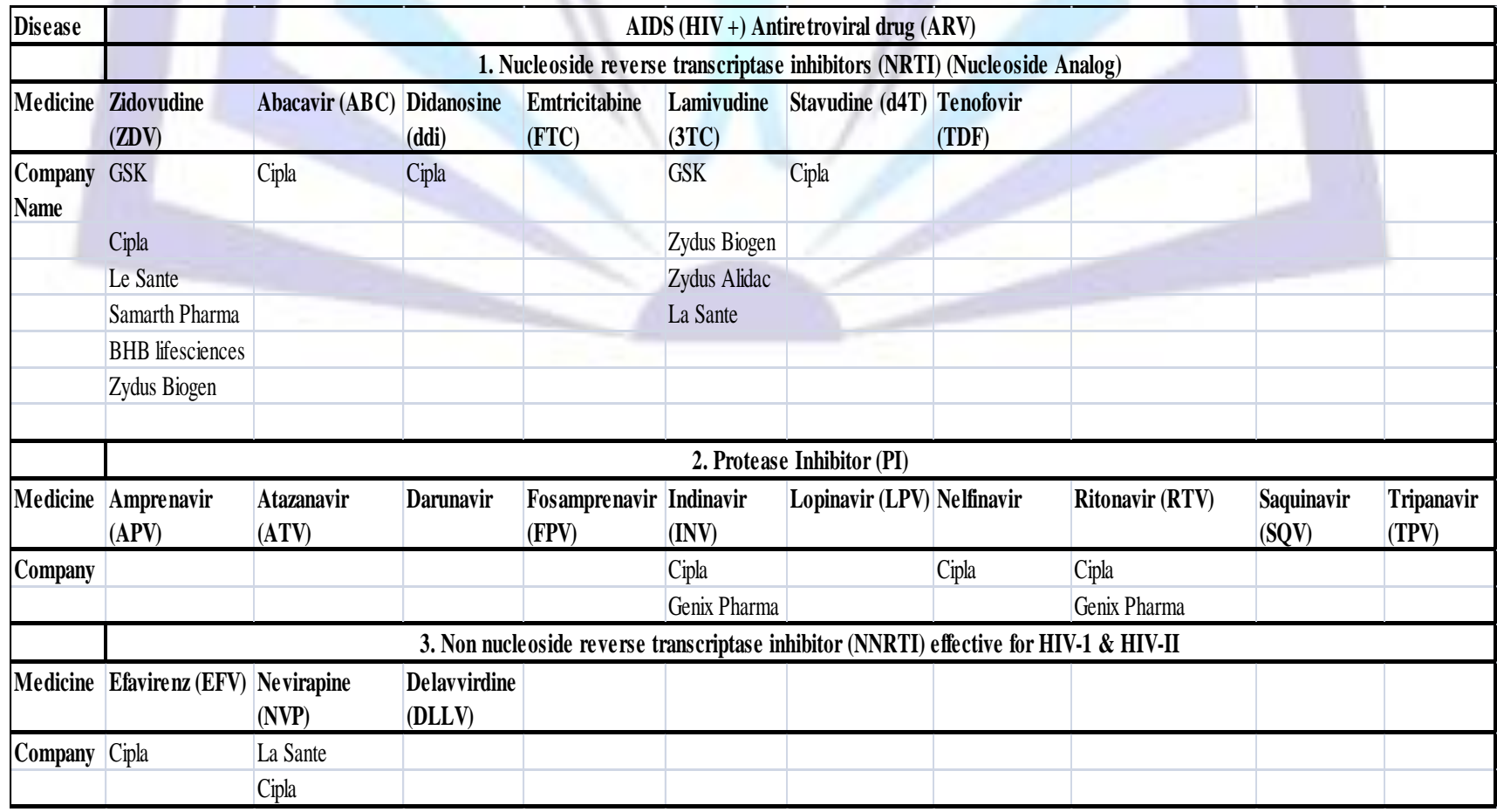


Table-4. 2nd Line drugs for Multi drug resistant Tuberculosis and company name

\begin{tabular}{|c|c|c|c|c|c|c|c|c|c|c|}
\hline \multirow{2}{*}{\begin{tabular}{|l|} 
Disease \\
Medicine
\end{tabular}} & \multicolumn{9}{|c|}{ Tuberculosis (2nd line drugs for Multi drug resistant Tuberculosis (MDRTB)) } & \multirow[b]{2}{*}{ Clofazimine } \\
\hline & Amikacin & Ciprofloxacin & Ofloxacin & Kanamycin & $\begin{array}{l}\text { Paraminosalicylic } \\
\text { acid (PAS) }\end{array}$ & Ethionamide & Prothionamide & Cycloserine & Capriomycin & \\
\hline \multirow{48}{*}{$\begin{array}{l}\text { Company } \\
\text { name }\end{array}$} & Cadilla Pharma & Sarabhai & Anichem Labs & Macleods & Macleods & Lupin & Le Sante & Macleods & Macleods & Atra Zeneca \\
\hline & NPIL & Alkem & Aquilla Labs & Samarth Pharma & Lupin & Overseas HC & Macleods & Lupin & & SPPL \\
\hline & Neon labs & Aquilla & Bestochem & Alembic & USV limited & Themes & Themis & BHB pharma & & \\
\hline & Biochem & Otsira genetica & Kee Pharma & & & Macleods & & Panacea & & \\
\hline & Comed & Bayer & Biochem & & & BHB Lifesciences & & Samarth & & \\
\hline & Lupin & NPIL & Biodrug & & & Le Sante & & & & \\
\hline & Indoco & Biochem & $\begin{array}{l}\text { Claris } \\
\text { Lifesciences }\end{array}$ & & & Panacea Biotech & & & & \\
\hline & Samarth Pharma & Plathico & Divus Labs & & & Samarth Pharma & & & & \\
\hline & Alkem & Blue Cross & Mimec pharma & & & & & & & \\
\hline & Indswift & Memec Pharma & Ethicare & & & & & & & \\
\hline & Plathico & Warren & Medico labs & & & & & & & \\
\hline & $\begin{array}{l}\text { Global Medical } \\
\text { sciences }\end{array}$ & Eglomed & Emcure & & & & & & & \\
\hline & Brown and Burk & Ranbaxy & Dey's & & & & & & & \\
\hline & Syntheko & Albert David & Neu Foreva & & & & & & & \\
\hline & Mapra Lab & Biological E & MarK remedies & & & & & & & \\
\hline & Aristo & Anichem Labs & Zenith HC & & & & & & & \\
\hline & Troikaa & Cipla & Merind & & & & & & & \\
\hline & Emcure & Indoco & Monochem & & & & & & & \\
\hline & Novamed & Core & e Merck & & & & & & & \\
\hline & Secure HC & Zydus Cadilla & Brussels & & & & & & & \\
\hline & Taurus & Emcure & Seagull Labs & & & & & & & \\
\hline & Cachet & Cadilla Pharma & Blue Cross & & $x^{2}$ & & & & & \\
\hline & & Glenmark & Khandelwal & & & & & & & \\
\hline & & Lark Labs & Concepts & & & & & & & \\
\hline & & Dr. Reddy's Labs & Oglamed & & & & & & & \\
\hline & & Lupin Pharma & Zydus Cadilla & & & & & & & \\
\hline & & Alembic & Macleods & & & & & & & \\
\hline & & $\begin{array}{l}\text { Claris } \\
\text { Lifesciences }\end{array}$ & Suniz Pharma & & & & & & & \\
\hline & & Mankind & Medley Pharma & & & & & & & \\
\hline & & Sun Pharma & Emcure & & & & 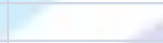 & & & \\
\hline & & GSK & Aventis & & & & & & & \\
\hline & & Macleods & Vales & & & & 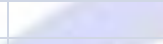 & 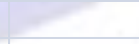 & & \\
\hline & & Concept & Indswift & 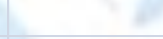 & 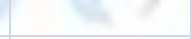 & & 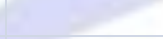 & & & \\
\hline & $\sin$ & Dey's & Taurus Lab & & & & & & & \\
\hline & 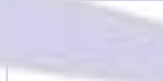 & $\begin{array}{l}\text { HAL (Hindustan } \\
\text { Antibiotic limited) }\end{array}$ & Balpharma & & & & & & & \\
\hline & & Formed & Mankind & & & & & & & \\
\hline & & $\begin{array}{l}\text { Karnataka } \\
\text { Antibiotic ltd } \\
\text { (KAPL) }\end{array}$ & FDC & & & & & & & \\
\hline & & NPIL & Stancare & & & & & & & \\
\hline & & Biddle Sawyer & & & & & & & & \\
\hline & & Torrent & & & & & & & & \\
\hline & & Dabur & & & & & & & & \\
\hline & & Hygeia & & & & & & & & \\
\hline & & Khandelwal & & & & & & & & \\
\hline & & Indswift & & & & & & & & \\
\hline & & Taurus & & & & & & & & \\
\hline & & Balpharma & & & & & & & & \\
\hline & & Wyeth Laderly & & & & & & & & \\
\hline & & FDC & & & & & & & & \\
\hline
\end{tabular}


ISSN 2278-5612

Table-5 - List of Oral Glycemic Agents used for treatment of Diabetes Mellitus

\begin{tabular}{|c|c|c|c|c|c|c|c|c|c|}
\hline \multirow[t]{2}{*}{ Disease } & \multicolumn{9}{|c|}{ Diabetes mellitus (Oral Hypoglycemic agents) } \\
\hline & & & & & 1. Sulphonyl ureas & & & & \\
\hline Medicine & Chloropropamide & Tolbutamide & Glibenclamide & Glipizide & Gliclazide & Glimepride & $\begin{array}{l}\text { Rosilglitazone } \\
+ \text { Glicazide }\end{array}$ & Rosilglitazone & Pioglitazone \\
\hline \multirow[t]{30}{*}{ Company } & Dey's Pharma & & Cadilla Pharma & Emcure & Torrent & Aventis & Aristo & Torrent (psycan) & Biochem \\
\hline & & & Zydus Medica & Bal Pharma & Comed & Torrent & & Dr. Reddy's Lab & Unisearch \\
\hline & & & Sarabhai & Microlabs & Bal Pharma & Panacea & & Sun Pharma & Medley \\
\hline & & & Aventis & Otsira Genetica & Serdia & BioChem & & Aristo & Indswift \\
\hline & & & Inga & Franco Indian & Carsyon & Microlabs & & Healtheon & Zydus Medica \\
\hline & & & NPIL & Modi Mundi Pharma & RPG Lifesciences & Emcure & & Microlabs & Panacea \\
\hline & & & Bal Pharma & RPG lifesciences & Argus & Medley & & RPG Lifesciences & Wockhardt \\
\hline & & & Sun Pharma & Alembic & Inga & Argus & & Emcure & Healtheon \\
\hline & & & USV & Wallace & Alkem & Comed & & & Systopic \\
\hline & & & Otsira Genetica & Jenburkt & Khandelwal & RPG Lifesciences & & & Ranbaxy \\
\hline & & & & USV & Modi Mundi Pharma & Otsira Genetica & & & Sun Pharma \\
\hline & & & & Stadmed & JKDPL & Ranbaxy & & & Emcure \\
\hline & & & & Dey's & Stadmed & Wallace & & & KAPL \\
\hline & & & & & Panacea & Sun Pharma & & & Alembic \\
\hline & & & & & Raptakos & Cadilla Pharma & & & Intas \\
\hline & & & & & Medley & Win Medicare & & & Life Medicare \\
\hline & & & & & Indoco & Kopran & & & Comed \\
\hline & & & & & Aristo & Bal Pharma & & & Karsyon \\
\hline & & & & & IPCA & Life Medicare & & & Balpharma \\
\hline & & & & & USV & Intas & & & Otsira Genetica \\
\hline & & & & & Protec & & & & Aglomed \\
\hline & & & & & Cadilla Pharma & & & & $\begin{array}{l}\text { RPG } \\
\text { Lifesciences }\end{array}$ \\
\hline & & & & & Novartis & & & & Kopran \\
\hline & & & & & Alembic & & & & Argus \\
\hline & & & 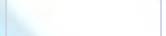 & & Jagsonpal & & & & IPCA \\
\hline & & & 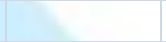 & & Dey's & 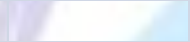 & 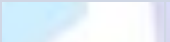 & & Indoco \\
\hline & & & 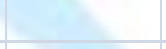 & & Intas & & . & & NPIL \\
\hline & & . & 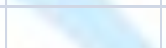 & & Dr.Reddy's labs & 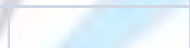 & 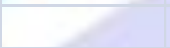 & & Franco Indian \\
\hline & & & & 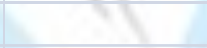 & & & 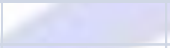 & & Cadilla Pharma \\
\hline & & & 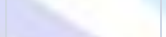 & nes & 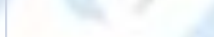 & 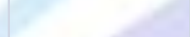 & 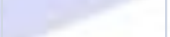 & & E.Merck \\
\hline
\end{tabular}

Table-6 List of company making Insulin for treatment of Diabetes

\begin{tabular}{|l|l|l|l|l|l|l|}
\hline Disease & \multicolumn{5}{|c|}{ Diabetes mellitus (Insulin) } \\
\hline Medicine & $\begin{array}{l}\text { Neutral Insulin } \\
\text { (Soluble) }\end{array}$ & $\begin{array}{l}\text { Isophane Insulin } \\
\text { (NPH) }\end{array}$ & $\begin{array}{l}\text { Insulin Zinc suspension } \\
\text { (Lente Insulin) }\end{array}$ & $\begin{array}{l}\text { Pre mixed } \\
\text { biphasic Insulin }\end{array}$ & Insulin Lispro & Insulin Glargine \\
\hline Company & Novo Nordisc & Biocon & Novo Nordisc & USV & Eli lily & Aventis Pharma \\
& USV & USV & Eli-Lily & Novo Nordisc & Ranbaxy & \\
\hline & Sarabhai & Novo Nordisc & Ranbaxy & Sara Bhai & & \\
\hline & Eli lily & Sara Bhai & Sarabhai & Eli-ily & & \\
\hline & Ranbaxy & Eli-lily & Cadilla Pharma & Ranbaxy & & \\
\hline & Aventis & Aventis & & Aventis & & \\
\hline & Cadilla Pharma & IRM Pharma & & Cadilla Pharma & & \\
\hline
\end{tabular}

(Source: Drug Today) 
Table-7 List of company making Biguanides for treatment of Diabetes

\begin{tabular}{|c|c|c|c|c|c|c|c|c|c|}
\hline & \multicolumn{9}{|c|}{ Biguanides and it's combinations } \\
\hline Medicine & Phenphormine & Metphormine & $\begin{array}{l}\text { Metphormine + } \\
\text { Glibenclamide }\end{array}$ & $\begin{array}{l}\text { Phenphormine } \\
+\end{array}$ & $\begin{array}{l}\text { Metphormine } \\
+ \text { Glipizide }\end{array}$ & $\begin{array}{l}\text { Metphormine + } \\
\text { Gliclazide }\end{array}$ & $\begin{array}{l}\text { Metphormine + } \\
\text { Glimpride }\end{array}$ & $\begin{array}{l}\text { Metphormine + } \\
\text { Rosiglitazone } \\
\end{array}$ & $\begin{array}{l}\text { Metphormine + } \\
\text { Pioglitazone }\end{array}$ \\
\hline Company & USV & Ostira Genetica & RPG Lifesciences & Cadilla Pharma & Nucron Pharma & Torrent & Novartis & Torrent & Unisearch \\
\hline & & Aventis & NPIL & & Bal Pharma & Comed & Torrent & Aztec & Stancare \\
\hline & & Argus & USV & & Novartis & Bal Pharma & Panacea & Aristo & Sunpharma \\
\hline & & CFL & Bal Pharma & & Microlabs & Carsyon & Otsira Genetica & Healtheon & Emcure \\
\hline & & Torrent & Sun Pharma & & Franco Indian & Argus & Cadilla Pharma & Panacea & Intas \\
\hline & & Bal pharma & Modi Mundi Pharma & & Aztec & Alkem & NPIL & Microlabs & Carsyon \\
\hline & & Novarts & Win Medicare & & Zenburkt & Biochem & Unisearch & Emcure & Otsira genetica \\
\hline & & Stadmed & NPIL & & USV & Khandelwal & Intas & & NPIL \\
\hline & & IPCA & Argus & & Dey's Pharma & Modi Mundi & & & Cadilla Pharma \\
\hline & & Dey's & & & Otsira Genetica & Win Medicare & & & \\
\hline & & Alkem & & & Taurus Labs & Stadmed & & & \\
\hline & & Alembic & & & Tridoss & Panacea & & & \\
\hline & & NPIL & & & & Raptakos & & & \\
\hline & & Protec & 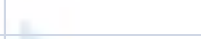 & & & Medlay Pharma & & & \\
\hline & & Franco Indian & 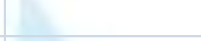 & & & Indoco & & & \\
\hline & & Emcure & & & & Aristo & & & \\
\hline & & Cadilla Pharma & & & & IPCA & & & \\
\hline & & Microlabs & & & & Cipla & & & \\
\hline & & Comed & & & & Mankind Pharma & & & \\
\hline & & Panacea & & & & Cadilla Pharma & & & \\
\hline & & Zenburkt & & & & Novartis & & & \\
\hline & & PIL & & & & Alembic & & & \\
\hline & & Ranbaxy & & & & Dey's & & & \\
\hline & & RPG lifesciences & & & & Jagsonpal & & & \\
\hline & & Wallace & & & & Intas & & & \\
\hline & & Healtheon & & & & Dr. Reddy Labs & & & \\
\hline & & Raptakos & & & & Wallace & & & \\
\hline & & Intas & & & & PIL & & & \\
\hline & & & & & Others & 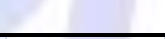 & & & \\
\hline Medicine & Acarbose & Repaglinide & Nateglinide & & 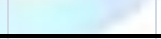 & & & & \\
\hline & Emcure & Torrent & Healtheon & & & & & & \\
\hline & Wockhardt & Azitec (Sun) & Alembic & & & & & & \\
\hline & Cardicare & Wallace & Intas & & & & 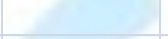 & & \\
\hline & Balpharma & & Emcure & & & 1 & & & \\
\hline & Otsira Genetica & & & & & 1 & & 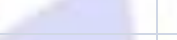 & \\
\hline & Healtheon & & & & & 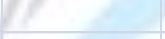 & & & \\
\hline & Medley & 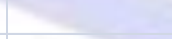 & s & . & 7 & & & & \\
\hline & Arian, Sun & & 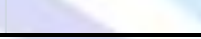 & & . & 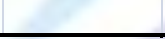 & 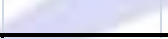 & & \\
\hline
\end{tabular}

From the above work we came across 87 pharmaceutical firms relevant for the study. The data was collected from the Annual report of companies and was also gathered from Bloomberg, Mar, 2013.

"Many companies manufacturing or distributing a range of products will find that the Pareto Law will apply and can be exploited to determine supply strategy. Typically an analysis of the business will show that the $80 / 20$ (or similar) rule holds. In other words, $80 \%$ of total volume will be generated from just $20 \%$ of the total product line."( Martin Christopher and Denis Towill (2001). Considering the above stated Pareto's approach we have considered 24 pharmaceutical firms which contribute to more than $80 \%$ of the business. We could get the data for 24 companies which are listed in stock exchange and for those companies whose websites were having the Annual report for the year 2012.

\section{Data Envelopment Analysis (DEA)}

Data Envelopment Analysis (DEA) is a non-parametric method of measuring efficiency of any Decision Making Unit (DMU) like firms, retail stores, public sector agency etc (Ramanathan, R. (2003). It was first introduced into the operation research by Charnes, Cooper and Rhodes in 1978 which is known as CCR model. This model has an assumption of constant returns to scale. Then, in 1984, Banker, Charnes and Cooper introduced a new model, known as BCC model. This model was an extension of the CCR model to accommodate the variable return to scale into consideration while analyzing the efficiency or performance of any DMU.

CCR model takes $n$ DMUs into consideration, using $m$ inputs to secure $s$ outputs. The notations which are used in the model are as follows-

m: number of inputs $(i=1,2, \ldots m)$ 
$\mathrm{s}$ : number of outputs $(r=1,2, \ldots \mathrm{s})$

$n$ : number of DMUs $(j=1,2, \ldots n)$

$x_{i j}: i^{\text {th }}$ input of $j^{\text {th }} D M U$

$y_{r j}: r^{\text {th }}$ output of $j^{\text {th }} D M U$

$\mathrm{X}=\left(\begin{array}{cccc}x_{11} & x_{12} & \ldots & x_{1 n} \\ x_{21} & x_{22} & \ldots & x_{2 n} \\ \cdot & \cdot & \ldots & \cdot \\ x_{m 1} & x_{m 2} & \ldots & x_{m n}\end{array}\right) \mathrm{Y}=\left(\begin{array}{cccc}y_{11} & y_{12} & \ldots & y_{1 n} \\ y_{21} & y_{22} & \ldots & y_{2 n} \\ \cdot & \cdot & \ldots & \cdot \\ y_{s 1} & y_{s 2} & \ldots & y_{s n}\end{array}\right)$

$\mathrm{u}=\left(\mathrm{u}_{1}, \mathrm{u}_{2}, \ldots . \mathrm{u}_{\mathrm{s}}\right) \quad \mathrm{v}=\left(\mathrm{v}_{1}, \mathrm{v}_{2}, \ldots . \mathrm{v}_{\mathrm{m}}\right)$

The efficiency of each DMU is measured once and hence we need $\mathrm{n}$ optimization problem to be solved, one for each $\mathrm{DMU}_{\mathrm{j}}$. Let the $\mathrm{DMU}_{\mathrm{j}}$ to be evaluated on any trial be designated as $\mathrm{DMU}_{\mathrm{o}}$, where "o"ranges over $1,2, \ldots ., \mathrm{n}$. We solve the following $L P$ to obtain values for the input weights $v_{1}, v_{2}, \ldots . v_{m}$ and output weight $u_{1}, u_{2}, \ldots . u_{s}$ as variables.

$\max \mathrm{u}_{1} \mathrm{y}_{10}+\mathrm{u}_{2} \mathrm{y}_{20}+\ldots \ldots+\mathrm{u}_{\mathrm{s}} \mathrm{y}_{\mathrm{so}}$

s.t. $\quad v_{1} x_{10}+v_{2} x_{20}+\ldots . .+v_{m} x_{m o}=1$

$$
\begin{aligned}
& u_{1} y_{1 j}+u_{2} y_{2 j}+\ldots .+u_{s} y_{s j} \leq v_{1} x_{1 j}+v_{2} x_{2 j}+\ldots \ldots+v_{m} x_{m j} \quad(j=1,2, \ldots ., n) \\
& v_{1}, v_{2}, \ldots . v_{m} \geq 0 \\
& u_{1}, u_{2}, \ldots . u_{s} \geq 0
\end{aligned}
$$

The dual of LP helps in recognizing the reference set for the inefficient DMUs. These reference sets then help us in identifying the inadequacies existing in the inefficient units. The dual of the above model can be given in the following form-

$\min \theta=\theta^{*}$

s.t. $\quad \mathrm{Y} \lambda \geq \mathrm{y}_{0}$

$X \lambda \leq \theta x_{0}$

$\lambda \geq 0$

The BCC model differs from the CCR model only in the adjunction of the condition $\sum_{j=1}^{n} \lambda_{j}=1$ which we also write $e \lambda=1$ where $\mathrm{e}$ is a row vector with all elements unity and $\lambda$ is a column vector with all elements non-negative.

The above model provides technical efficiency $(\theta)$ of any DMU.

Phase -1

$\operatorname{Min} \theta=\theta^{*}$

s.t. $\quad Y \lambda \geq y_{0}$

$X \lambda \leq \theta x_{0}$

$\mathrm{e} \lambda=1$

$\lambda \geq 0$

We solve phase-I to get $\theta^{*}$. Then using our knowledge of $\theta^{*}$, we solve phase-Il.

Phase-II

$\operatorname{Min} \sum \mathrm{s}_{\mathrm{i}}^{-}-\sum \mathrm{s}_{\mathrm{r}}^{+}$

s.t.

$\mathrm{Y} \lambda-\mathrm{s}_{\mathrm{r}}^{+}=\mathrm{yr}_{0}, \quad \mathrm{r}=1,2,3, \ldots \mathrm{s}$

$X \lambda+s_{i}{ }^{-}=\theta^{*} x_{i 0}, i=1,2,3, \ldots . m$

$\mathrm{e} \lambda=1$

$\lambda \geq 0, \mathrm{~s}_{\mathrm{i}}^{-}, \mathrm{s}_{\mathrm{r}}{ }^{+} \geq 0$ 


\section{Data Variables}

The variables selected for the study are categorized as input-output in the following manner

\section{Inputs}

The inputs considered are Expenditure on Research and development (R\&D), Cost of Materials, Weighted Average cost of capital (WACC).

To state, "Without a dramatic increase in R\&D productivity, today's pharmaceutical industry cannot sustain sufficient innovation to replace the loss of revenues due to patent expirations for successful products." (Paul et.al, 2010,). Expenditure on Research and Developments (R\&D) is needed for innovation and considered as a key input.

Cost of materials $(\mathrm{CM})$ is the cost of the raw materials consumed to make the finished goods.

The cost of capital is a standard tool of practical finance, so it's worth writing out the formula. Let $r_{D}$, and $r_{E}$, be the cost of debt and the cost of equity-that is, the expected rates of return demanded by investors in the firm's debt and equity securities. The overall (weighted-average) cost of capital depends on these costs and the market-value ratios of debt and equity to overall firm value.

Weighted Average Cost of Capital $=r_{A},=r_{D}, D / V+r_{E}, E / V$

The weighted average cost of capital $\mathrm{r}_{\mathrm{A}}$, is the expected return on a portfolio of all the firm's outstanding securities. (Myers, 2001).

A calculation of a firm's cost of capital in which each category of capital is proportionately weighted. All capital sources common stock, preferred stock, bonds and any other long-term debt - are included in a WACC calculation. All else equal, the WACC of a firm increases as the beta and rate of return on equity increases, as an increase in WACC notes a decrease in valuation and a higher risk. The WACC equation is the cost of each capital component multiplied by its proportional weight and then summing:

Weighted Average Cost Of Capital (WACC)

$\mathrm{WACC}=\frac{\mathrm{E}}{\mathrm{V}} * \mathrm{Re}+\frac{\mathrm{D}}{\mathrm{V}} * \mathrm{Rd} *(1-\mathrm{TC})$

Where:

$R e=$ cost of equity, $R d=$ cost of debt, $E=$ market value of the firm's equity, $D=$ market value of the firm's debt,

$\mathrm{V}=\mathrm{E}+\mathrm{D}, \mathrm{E} / \mathrm{V}=$ percentage of financing that is equity, $\mathrm{D} / \mathrm{V}=$ percentage of financing that is debt, Tc = corporate tax rate

Businesses often discount cash flows at WACC to determine the Net Present Value (NPV) of a project, using the formula: NPV = Present Value (PV) of the Cash Flows discounted at WACC.

\section{Outputs}

Outputs are Sales in million INR, Net Profit, Earning per Share (EPS), Export \%

Earnings per share (EPS) is the amount of earnings per each outstanding share of a company's stock. The portion of a company's profit allocated to each outstanding share of common stock. Earnings per share serves as an indicator of a company's profitability.

$$
\text { Earnings per share }(E P S)=\frac{\text { Net Income }- \text { Dividends on preferred stock }}{\text { Average outstanding Share }}
$$

When calculating, it is more accurate to use a weighted average number of shares outstanding over the reporting term, because the number of shares outstanding can change over time. However, data sources sometimes simplify the calculation by using the number of shares outstanding at the end of the period. Diluted EPS expands on basic EPS by including the shares of convertibles or warrants outstanding in the outstanding shares number.

We are dropping Sales from output as Sales and Profit are highly correlated as stated in Table-8.

Table-8. Correlations of outputs

\begin{tabular}{|l|c|c|c|c|}
\hline & O1 Sales & O2 Profit & O3 Earning / Share & O4 Export \\
\hline O1 Sales & 1 & & & \\
\hline O2 Profit & $.776^{* *}$ & 1 & & \\
\hline O3 Earning / Share & .201 & .219 & 1 & \\
\hline O4Export & .040 & .009 & .007 & 1 \\
\hline
\end{tabular}


The descriptive statistics for the outputs and inputs considered are presented in Table 9. The efficiency of DMUs, their peers, possible reductions of inputs in percentage, possible increase in outputs in percentage are presented in Table 10.

Table-9 Descriptive Statistics of outputs and inputs

\begin{tabular}{|l|r|r|r|r|r|}
\hline & $\mathrm{N}$ & Minimum & Maximum & Mean & Std. Deviation \\
\hline Profit & 24 & 9 & 25873 & 4866.75 & 5802.790 \\
Earning per Share & 24 & .51 & 76.76 & 26.0029 & 19.99184 \\
Export & 24 & 2.73 & 86.90 & 33.0217 & 21.15355 \\
R\&D & 24 & 3 & 6243 & 1591.67 & 1920.518 \\
CM & 24 & 314 & 23264 & 9167.79 & 7705.955 \\
WACC & 24 & 6.34 & 10.82 & 9.3254 & .86017 \\
Valid N (listwise) & 24 & & & & \\
\hline
\end{tabular}

Table 10 Efficiencies, Peers and Projections

\begin{tabular}{|c|c|c|c|c|c|c|c|c|}
\hline \multirow{2}{*}{\multicolumn{3}{|c|}{ Phase -I }} & \multicolumn{6}{|c|}{ Phase-II } \\
\hline & & & \multicolumn{3}{|c|}{ Output $\%$ to increase } & \multicolumn{3}{|c|}{ Input $\%$ to decrease } \\
\hline DMU\# & BCC effi. & $\begin{array}{l}\text { Benchmark } \\
\text { DMU \#s }\end{array}$ & Net Profit (02) & $\begin{array}{l}\text { EPS (earning per } \\
\text { share) (03) }\end{array}$ & Export \% (04) & R\&D Spent (I1) & $\begin{array}{l}\text { Cost of Material, } \\
\operatorname{cogs}(12)\end{array}$ & $\begin{array}{l}\text { Weighted Average cost } \\
\text { of capital WACC (\%) (I3) }\end{array}$ \\
\hline 1 & 0.73 & $8,13,19$ & 107 & 538 & 0 & 53 & 27 & 27 \\
\hline 2 & 0.85 & $13,19,21$ & 23 & 0 & 360 & 15 & 36 & 15 \\
\hline 3 & 0.75 & $8,19,22$ & 0 & 151 & 0 & 61 & 46 & 25 \\
\hline 4 & 0.87 & $8,19,22$ & 0 & 28 & 0 & 76 & 47 & 12 \\
\hline 5 & 0.98 & $8,19,22$ & 0 & 153 & 0 & 50 & 55 & 1.5 \\
\hline 6 & 0.94 & 13,21 & 6 & 111 & 21 & 5 & 84 & 5 \\
\hline 7 & 1 & & 0 & 0 & 0 & 0 & 0 & 0 \\
\hline 8 & 1 & & 0 & 0 & 0 & 0 & 0 & 0 \\
\hline 9 & 1 & & 0 & 0 & 0 & 0 & 0 & 0 \\
\hline 10 & 0.89 & 13,19 & 19 & 0 & 239 & 11 & 64 & 11 \\
\hline 11 & 1 & & 0 & 0 & 0 & 0 & 0 & 0 \\
\hline 12 & 0.76 & $8,19,22$ & 0 & 152 & 0 & 74 & 66 & 23 \\
\hline 13 & 1 & & 0 & 0 & 0 & 0 & 0 & 0 \\
\hline 14 & 0.9 & $13,19,21$ & 118 & 547 & 0 & 9 & 64 & 9 \\
\hline 15 & 0.91 & 8,19 & 43 & 92 & 0 & 56 & 34 & 8 \\
\hline 16 & 0.72 & 8,19 & 258 & 74 & 0 & 76 & 42 & 27 \\
\hline 17 & 1 & & 0 & 0 & 0 & 0 & 0 & 0 \\
\hline 18 & 0.94 & $8,19,22$ & 0 & 98 & 0 & 79 & 53 & 5 \\
\hline 19 & 1 & & 0 & 0 & 0 & 0 & 0 & 0 \\
\hline 20 & 0.85 & 19,22 & 0 & 76 & 0 & 43 & 60 & 14 \\
\hline 21 & 1 & & 0 & 0 & 0 & 0 & 0 & 0 \\
\hline 22 & 1 & & 0 & 0 & 0 & 0 & 0 & 0 \\
\hline 23 & 0.81 & $8,19,22$ & 0 & 30 & 0 & 73 & 38 & 18 \\
\hline 24 & 0.85 & & 0 & 38 & 0 & 82 & 64 & 14 \\
\hline
\end{tabular}

In order to clearly examine the determinants of efficiency, we further applied Tobit regression model regressing the BCC efficiency (as shown in second column of Table 10) as dependent variable. As per DEA literature Coelli(1998), Tobit regression model is suitable when the dependent variable is censored. The Tobit regression model is represented as-

$$
\begin{aligned}
\theta_{i}= & \alpha_{i}+\beta_{1}(\operatorname{Pr} \text { ofit })_{i}+\beta_{2}(\text { EPS })_{i}+\beta_{3}(\text { Export })_{i}+\beta_{4}(R \& D)_{i}+\beta_{5}(C M)_{i} \\
& +\beta_{6}(\text { WACC })_{i}+\varepsilon_{i}
\end{aligned}
$$

Where, $\theta_{i}$ is the efficiency score for the pharmaceutical company, $i$ computed from the BCC model.

The BCC efficiency statistics are as shown below. 
Table 11: BCC efficiency statistics

\begin{tabular}{|l|c|c|c|c|}
\hline & Minimum & Mean & Maximum & SD \\
\hline BCC efficiency & 0.72 & 0.9062 & 1.0000 & 0.09753 \\
\hline
\end{tabular}

The $\chi^{2}$ test statistics $(=244.8)$ with five degrees of freedom associated with $\mathrm{p}$ value $(=0.0)$ shows that the model is a good fit for the data. Also we find that the value of constant $2\left(e^{-2.52}=0.08046\right)$ from the Tobit model is less that the standard deviation of $\theta_{i}(=0.09753)$ which again shows that the models appears to fit the data well. The results of Tobit regression are presented in Table 12.

Table 12: Tobit regression model

\begin{tabular}{|l|c|r|r|r|}
\hline \multicolumn{1}{|c|}{ Variable } & \multicolumn{1}{c|}{ Value } & \multicolumn{1}{c|}{ Std. Error } & z-value & p-value \\
\hline Constant & 1.6600000 & 0.27800000 & 5.95493 & \\
\hline Profit* & 0.0000432 & 0.00001680 & 2.57840 & 0.004963 \\
\hline Earning per Share & 0.0003140 & 0.00134000 & 0.23538 & 0.406957 \\
\hline Export* & -0.0022300 & 0.00123000 & 1.80903 & 0.035223 \\
\hline R\&D** & -0.0000519 & 0.00001745 & -2.98381 & 0.004123 \\
\hline CM $^{* *}$ & -0.0000112 & 0.00000701 & -1.60411 & 0.054345 \\
\hline WACC $^{* *}$ & -0.0086300 & 0.03030000 & -2.84309 & 0.002234 \\
\hline
\end{tabular}

** Significant

\section{Recommendations and conclusions}

DEA was useful to find out the benchmark DMU / companies. It was found that all companies are above $70 \% \mathrm{BCC}$ efficient. The benchmark companies for each are cited in Table 10. There is a tremendous scope to reduce the R\&D spent. It can be inferred that even though the spent on R\&D is more but it is not yielding enough. The cost of materials also needs to be reduced. The inference is that these firms are using cost plus margin system and there is good scope for backward negotiations with the vendor to reduce the cost of raw materials which can help society in large to produce drugs at affordable prices. The results derived through Tobit regression analysis highlights that the efficiency of pharmaceutical firms is mainly impacted either by output variables namely profit and export or by input variables i.e. R\&D and WACC.

\section{REFERENCES}

[1] Banker, R. D., Charnes, A. and Cooper, W.W. (1984) "Some Models for Estimating Technical and Scale Inefficiencies in Data Envelopment Analysis," Management Science, No. 30, pp.1078-1092

[2] Banker R D and Morey R C (1986). Efficiency analysis for exogenously fixed inputs and outputs, Management Science, 43, 4, 513-521.

[3] Charnes, A., Cooper, W.W. and Rhodes, E. (1978), "Measuring the Efficiency of Decision Making Units", European Journal of Operational Research, Vol. 2, 1978, pp.429-444.

[4] Chaudhuri S (1999)."Growth and Structural Changes in the Pharmaceutical Industry in India" in Sen Anindya, Gokarn Subir and Vaidya Rajendra (eds), The Structure of Indian Industry, Oxford University Press, New Delhi.

[5] Chia-Chien Hsu, Brian A. Sandford "The Delphi Technique: Making Sense Of Consensus", Practical Assessment, Research \& Evaluation, Vol 12, No 10 August 2007 ISSN 1531-7714

[6] Coelli, T.J., Rao, P. And Battese, G.E. (1998), An Introduction to Efficiency and Productivity Analysis, Kluwer Academic Press, Dordrecht

[7] Crofton John, Pierre Chaulet \& Maher Dermont - Guidelines for the management of drug- resistant Tuberculosis WHO,Geneva,1997. 
[8] Dhar Biswajit, and Gopakumar K.M.,(2006) "Post-2005 TRIPS scenario in patent protection in the pharmaceutical sector: The case of the generic pharmaceutical industry in India" , UNCTAD/ICTSD Project on Intellectual Property Rights and Sustainable Development

[9] Dalkey, N. C. (1972). The Delphi method: An experimental study of group opinion. In N. C. Dalkey, D. L. Rourke, R. Lewis, \& D. Snyder (Eds.). Studies in the quality of life: Delphi and decision-making (pp. 13-54). Lexington, MA: Lexington Books.

[10] Dalkey, N. C., \& Helmer, O. (1963). An experimental application of the Delphi method to the use of experts. Management Science, 9 (3), 458-467.

[11] Drug Today (2012), January - March 2012, Vol.No.1 \& II.

[12] FICCl - Ernst \& young study (2010)

[13] Gene Rowea, George Wrightb (1999) "The Delphi technique as a forecasting tool: issues and analysis" International Journal of Forecasting 15 (1999) 353-375

[14] Harries Anthony, Maher Dermont \& Uplekar Mukund, TB-A clinical manual for South -east Asia:World Health Organisation, Geneva,1997.

[15] Industry Drug Review (2007), A Mediworld publication, Nov-Dec (2007)

[16] Kiran Ravi \& Mishra Sunita (2011) "Research and Development, Exports and Patenting in the Indian Pharmaceutical Industry: a Post TRIPS Analysis," Eurasian Journal of Business and Economics 2011, 4 (7), 53 67.

[17] Kotwani Anita, Ewen Margaret, Dey Dalia, Iyer Shobha, P.K. Lakshmi, Patel Archana, Raman Kannamma Singhal G.L., Thawani Vijay, Tripathi Santanu \& Laing Richard, (2007) "Prices \& availability of common medicines at six sites in India using a standard methodology"Indian J Med Res 125, May 2007, pp 645-654

[18] Malhotra Rashmi and Malhotra D.K., (2008) "Analyzing the Performance of Pharmaceutical Firms Using Data Envelopment Analysis”, Sixth AIMS International Conference on Management, December 28-31, 2008

[19] Martin Christopher and Denis Towill, 2001, An Integrated Model for the Design of Agile Supply Chains, International Journal of Physical Distribution and Logistics Management, Vol. 31, No. 4, pp235-246, 2001

[20] Mazumdar Mainak, Meenakshi Rajeev, Ray Subhash C., (2010) "Sources of Heterogeneity in the Efficiency of Indian Pharmaceutical Firms" Centre for Social Sciences and Humanities occasional paper n²7/2010.

[21] Myers Stewart C., 2001, Capital Structure, The Journal of Economic Perspectives, Vol. 15, No. 2. (Spring, 2001), pp. 81-102.)

[22] News papers : Times Of India (TOI), News report:16.02.2011, Mergers \& Acquisitions in Pharmaceutical industry-: "Pharma sector injects fresh life into M \&A space, TOI dt.(16.02.2011)-" New Ingredients in drugs? Can't retail retain old name") , Economic Times, 1st March 2012

[23] Pannu,H.S., Dinesh Kumar, U. and Farooquie,J.A., (2010)," Impact of Innovation on the Performance of Indian Pharmaceutical Industry using Data Envelopment Analysis", IIMB working paper no.2010-02-302

[24] Paul Steven M., Daniel S. Mytelka, Christopher T. Dunwiddie, Charles C. Persinger, Bernard H. Munos, Stacy R. Lindborg and Aaron L. Schacht (2010), "How to improve R\&D productivity: the pharmaceutical industry's grand challenge"NATURE REVIEWS | Drug Discovery Volume 9 | March 2010 | 203, Macmillan Publishers Limited.

[25] Sampath Padmashree Gehl (2005) "Economic Aspects of Access to Medicines after 2005: Product Patent Protection and Emerging Firm Strategies in the Indian Pharmaceutical Industry" United Nations UniversityInstitute for New Technologies (UNU-INTECH) Study commissioned by the CIPIH, WHO.

[26] Saranga, Haritha and Phani, B.V (2004), "The Indian Pharmaceutical Industry - An Overview of Internal Efficiencies using Data Envelopment Analysis", Social Science Research Network, Working Paper Series, Rochester

[27] Saranga,.H. (2007) "Multiple objective data envelopment analysis as applied to the Indian Pharmaceutical Industry" Journal of the Operational Research Society (2007) 58, $1480-1493$

[28] Sengupta, Amit (2010) "Study of National Health System in India with regards Access to Health Care and Medicines," National Campaign Committee for Drug Policy, New Delhi, India April,2010

[29] Ramanathan, R. (2003), "An introduction to Data Envelopment Analysis: A Tool for Performance Mesurement", Sage Publications, New Delhi.

[30] Ray, S.C (2004). "Data Envelopment Analysis: Theory and Techniques for Economics and Operations Research", Cambridge University Press, chapters 1-3. pp 1-81.

Websites:

[31] www.bloomberg.com/ 
[32] www.cdc.gov/ncidod/eid/vol1no1/morse.htm,

[33] www.contractpharma.com/articles/2007/07/2007-top-20-pharmaceutical-companies-report

[34] www.fdli.org/pubs/journal/toc/vol54_2.html

[35] www.medindia.net/health_statistics/diseases/diabetes.asp,www.milbank.org/reports/0601fauci/0601fauci.html\#ba sic

[36] www.moneyexpress.com/business/33701.txthtml, www.pharmaceuticals.gov.in,

[37] www.rediff.com/money/2005/jan/html, www.who.int/evidence/en/

\section{Author' biography with Photo}

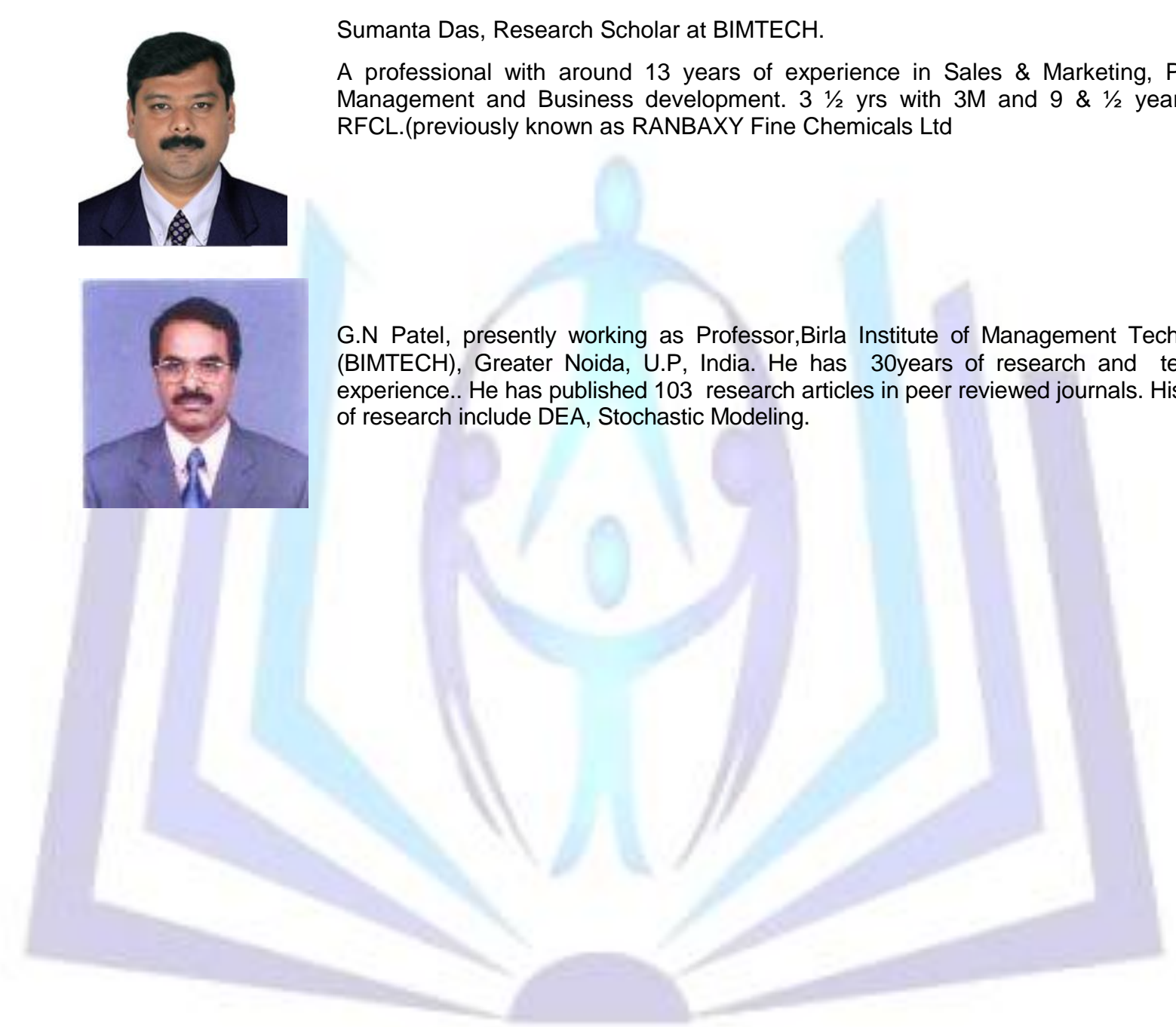

